\title{
Aurora kinase A (AURKA) and never in mitosis gene A-related kinase 6 (NEK6) genes are upregulated in erosive esophagitis and esophageal adenocarcinoma
}

\author{
ELMAS KASAP ${ }^{1}$, SEDA ÖRENAY BOYACIOGLU ${ }^{2}$, MEHMET KORKMAZ $^{3}$, ELIF SARITAS YUKSEL ${ }^{4}$, \\ BELKIS ÜNSAL $^{4}$, ERKAN KAHRAMAN ${ }^{3}$, ÖMER ÖZÜTEMIZ ${ }^{5}$ and HAKAN YUCEYAR ${ }^{1}$ \\ Departments of ${ }^{1}$ Gastroenterology, ${ }^{2}$ Medical Genetics and ${ }^{3}$ Medical Biology, Medical Faculty, \\ Celal Bayar University, Manisa; ${ }^{4}$ Department of Gastroenterology, Ataturk Research and Training Hospital, \\ Izmir; ${ }^{5}$ Department of Gastroenterology, Medical Faculty, Ege University, Izmir, Turkey
}

Received February 14, 2012; Accepted April 19, 2012

DOI: $10.3892 /$ etm.2012.561

\begin{abstract}
Gastroesophageal reflux disease is a risk factor for esophageal adenocarcinoma yet studies that have investigated the relationship between erosive esophagitis and esophageal adenocarcinoma have usually focused on symptom-related evidence or polymorphisms. There are no epigenetic gene expression studies on this topic. In this study, we aimed to evaluate the relationship between erosive esophagitis and esophageal adenocarcinoma to identify whether there is a genetic predisposition for esophageal adenocarcinoma. The Human Epigenetic Chromatin Modification Enzyme RT ${ }^{2}$ Profiler $^{\mathrm{TM}}$ PCR array (PAHS-085A) was used to detect the expression of $84 \mathrm{key}$ genes encoding enzymes. This was carried out prospectively for samples from 60 patients (20 patients as a control group, 20 patients with erosive esophagitis and 20 patients with esophageal adenocarcinoma). AURKA, AURKB, NEK6 were expressed at significantly higher levels in esophageal adenocarcinoma compared to the control group. MBD2 was expressed at significantly lower levels in the esophageal adenocarcinoma group compared to the control group. AURKA, AURKC, HDAC9 and NEK6 were expressed at significantly higher levels in erosive esophagitis compared to the control group. There was no difference in upregulated gene expression between the erosive esophagitis
\end{abstract}

Correspondence to: Dr Elmas Kasap, Department of Gastroenterology, Faculty of Medicine, Celal Bayar University, Manisa, Turkey

E-mail: elmaskasap@yahoo.com

Abbreviations: AURKA, aurora kinase A; AURKB, aurora kinase B; AURKC, aurora kinase C; BE, Barrett's esophagus; EAC, esophageal adenocarcinoma; EE, erosive esophagitis; GERD, gastroesophageal reflux disease; HDAC9, histone deacetylase 9; MBD2, methyl-CpG binding domain protein 2; NEK6, never in mitosis gene A-related kinase 6

Key words: aurora kinase A, never in mitosis gene A-related kinase, esophageal adenocarcinoma, erosive esophagitis and esophageal adenocarcinoma. MBD2 was significantly downregulated in esophageal adenocarcinoma compared to erosive esophagitis. NEK6 and AURKA were significantly upregulated in esophageal adenocarcinoma and erosive esophagitis compared to the control group. This is a novel study on the genetic predisposition for erosive esophagitis and esophageal adenocarcinoma. AURKA and NEK6 are two promising genetic markers for erosive esophagitis and esophageal adenocarcinoma.

\section{Introduction}

Esophageal cancer is the sixth most leading cause of cancer-related mortality worldwide (1). Esophageal adenocarcinoma (EAC) and esophageal squamous cell carcinoma are the two main histological types of esophageal cancers (1). Of all esophageal cancer types in Western countries, 30-50\% of cases are esophageal adenocarcinoma (1). The incidence of EAC has increased faster than that of any malignancy in Western countries, with an increase of $400 \%$ over the past 40 years (2). However, there has been no increase in the prevalence of proximal gastric cancers and distal esophageal adenocarcinomas in the Turkish population (3). The prognosis for EAC is poor and the overall 5-year survival rate is less than $10 \%$ (4). Risk factors for EAC include dietary factors, alcohol and tobacco use, obesity, gastroesophageal reflux disease and Barrett's esophagus (BE) (5). Gastroesophageal reflux disease (GERD) is very common worldwide (6). The prevalence of GERD is high, especially in developed Western countries (7). Bor et al found the prevalence of GERD to be $20 \%$ in Izmir (8) and $22.8 \%$ in Turkey, similar to the rates in the US (7). Chronic GERD is one of the main risk factors for the development of Barrett's esophagus (BE) and BE is one of the strongest risk factors for $\operatorname{EAC}(5,9)$. Mechanisms that control chromatin stucture and gene expression in normal mammalian cells are DNA methylation, covalent histone modifications, nucleosome position, histone variants and miRNAs (10-12). Recent epigenetic studies have shown the effect of epigenetic alterations in carcinogenesis as well as genetic alterations. A number of studies suggest that epigenetic alterations may even be initi- 
ating factors for certain types of cancer (13). Genetic alterations are irreversible but epigenetic alterations are reversible and this fact supports future hope for epigenetic therapy (14).

Studies that have investigated the relationship between erosive esophagitis (EE) and EAC usually focus on symptomrelated evidence or on polymorphisms. There are no epigenetic gene expression studies on this topic. We aimed to evaluate the relationship between EE and EAC to ascertain whether there is a genetic tendency for EAC.

\section{Materials and methods}

Location of study. The study was conducted at the Department of Gastroenterology, Department of Medical Biology, Celal Bayar University, Manisa between March 2010 and September 2011. Patients were also referred from the Department of Gastroenterology, Celal Bayar University, the Department of Gastroenterology, Ege University and the Department of Gastroenterology, Ataturk Research and Training Hospital.

Ethics. This study was performed in accordance with the Declaration of Helsinki, good clinical practice and applicable regulatory requirements. Celal Bayar University Institutional Review Board approved this clinical trial on June 2, 2009. Each patient signed a consent form prior to any study-related procedure.

Study design and subjects. Between March 2010 and September 2011 fresh paired tissue samples from 60 patients [group 1 (20 patients) categorized as the macroscopic and histopathologically confirmed esophageal carcinoma group; group 2 (20 patients) categorized as the erosive esophagitis (without histopathologically esophageal adenocarcinoma and Barrett's esophagus) group; and group 3 (20 patients) categorized as the control group (who had normal esophageal mucosa with no endoscopic or histopathological lesions)] were collected. Typical GERD symptoms were defined as at least five years of regurgitation and/or heartburn per week in erosive esophagitis. Patients were excluded from the study if they had a history of upper gastrointestinal surgery such as gastrectomy, fundoplication or distal esophagectomy, severe gastroparesis and esophageal varices.

\section{Endoscopy}

Erosive esophagitis and control group. Esophagogastroduodenoscopies were undertaken for the EE and control group at the Department of Gastroenterology, Celal Bayar University by the same two endoscopists (E.K., H.Y.) who performed the study. During upper gastrointestinal endoscopy, the distal $5 \mathrm{~cm}$ of the esophagus mucosal morphology at the squamo-columnar junction was visualized using conventional endoscopy followed by the Narrow Band Imaging (NBI) system using video endoscopes. During standard white-light endoscopy and NBI examination, erosions, mucosal breaks and other complications were graded according to the Los Angeles classification (15). Two biopsies were taken $2 \mathrm{~cm}$ above the esophagogastric junction from patients in the control group, and two biopsies were taken from mucosal breaks in patients with EE.
Esophageal adenocarcinoma. Esophagogastroduodenoscopies were undertaken for EAC cases at the Department of Gastroenterology, Celal Bayar University (11 patients), Department of Gastroenterology, Ege University and Department of Gastroenterology (5 patients), Ataturk Research and Training Hospital (4 patients). Two biopsies were taken from patients pathologically diagnosed as having EACs using Olympus biopsy forceps.

EAC was evaluated according to thoracic and abdominal computed tomography (CT) in three stages: stage 1, esophageal adenocarcinoma located in the esophagus; stage 2, esophageal adenocarcinoma located in the esophagus and with pathological lymphadenopathy; stage 3, esophageal adenocarcinoma located in the esophagus with pathological lymphadenopathy and distant metastasis.

Samples were immediately frozen using dry ice (a block of dry ice has a surface temperature of $-78.5^{\circ} \mathrm{C}$ ) and stored at $-80^{\circ} \mathrm{C}$ until RNA extraction.

Isolation of total RNA. Total RNA was extracted using the TriPure solution as described in the manufacturer's protocol. The fresh tissue was resuspended in a vial of MagNA Lyser Green Beads containing $350 \mu \mathrm{l}$ lysis buffer and $50 \mu \mathrm{l}$ of proteinase (Roche). Next, the suspension was subjected to mechanical lysis in a MagNA lyser instrument (Roche) for $45 \mathrm{sec}$ at $4500 \mathrm{rpm}$. Afterwards, RNA was further extracted and purified using a MagNA Pure LC instrument (Roche) in combination with the MagNA Pure NA isolation kit III. All steps were taken according to the manufacturer's protocol.

Quantity and purity of total RNA. RNA was quantified measuring the absorbance at $260 \mathrm{~nm}$ (A260 nm) and RNA purity was determined by the ratio A260 nm/A280 nm using a classical spectrophotometer. RNA quality was good, with 260/280 ratios slightly higher than 2.0 and 260/230 ratios slightly higher than 1.8 .

$R T^{2}$ profiler $^{T M} P C R$ protocol first strand cDNA synthesis. The protocol took $2 \mathrm{~h}$ to perform (per sample) from start to finish. We initially had as little as $25 \mathrm{ng}$ of total RNA from our experimental samples. We first converted the experimental RNA samples into PCR templates to prepare cDNAs with the RT ${ }^{2}$ First Strand kit (SABioscience, Frederick, MD, USA) according to the manufacturer's instructions. Next we combined the template with a specific instrument and used ready-to-use $\mathrm{RT}^{2} \mathrm{SYBR}$ Green qPCR Master Mix. Then we added equal aliquots of this mixture ( $25 \mu 1$ for 96 -well) to each well of the same PCR array plate containing the predispensed gene-specific primer sets and performed PCR. Specialized software (SABiosciences) was used to calculate the threshold cycle $(\mathrm{Ct})$ values for the genes on each PCR array.

Epigenetic chromatin modification enzyme PCR array. The Human Epigenetic Chromatin Modification Enzyme RT ${ }^{2}$ Profiler $^{\mathrm{TM}}$ PCR Array (PAHS-085A) (SABiosciences) was used to detect the expression levels of 84 key genes (Table I) encoding enzymes known or predicted to modify genomic DNA and histones to regulate chromatin accessibility and therefore gene expression. These genes exhibit differential expression profiles in tumor cells relative to normal cells. The 
Table I. List of key genes.

\begin{tabular}{|c|c|c|c|}
\hline Name & Description & Name & Description \\
\hline KDM1A & Lysine (K)-specific demethylase 1A & SETDB1 & SET domain, bifurcated 1 \\
\hline \multirow[t]{2}{*}{ ASH1L } & Ash1 (absent, small, or homeotic)-like & SETDB2 & SET domain, bifurcated 2 \\
\hline & (Drosophila) & SMYD3 & SET and MYND domain containing 3 \\
\hline ATF2 & Activating transcription factor 2 & KDM5C & Lysine (K)-specific demethylase 5C \\
\hline AURKA & Aurora kinase A & KDM4A & Lysine $(\mathrm{K})$-specific demethylase $4 \mathrm{~A}$ \\
\hline AURKB & Aurora kinase B & KDM4C & Lysine $(\mathrm{K})$-specific demethylase $4 \mathrm{C}$ \\
\hline AURKC & Aurora kinase $\mathrm{C}$ & KDM6B & Lysine $(\mathrm{K})$-specific demethylase 6B \\
\hline \multirow[t]{2}{*}{ CARM1 } & Coactivator-associated arginine & KAT2A & $\mathrm{K}$ (lysine) acetyltransferase $2 \mathrm{~A}$ \\
\hline & methyltransferase 1 & КАТ2B & $\mathrm{K}$ (lysine) acetyltransferase $2 \mathrm{~B}$ \\
\hline CDYL & Chromodomain protein, Y-like & KAT5 & $\mathrm{K}($ lysine $)$ acetyltransferase 5 \\
\hline \multirow[t]{2}{*}{ CIITA } & Class II, major histocompatibility complex, & MBD2 & Methyl-CpG binding domain protein 2 \\
\hline & transactivator & \multirow[t]{2}{*}{ MLL } & Myeloid/lymphoid or mixed-lineage leukemia \\
\hline CSRP2BP & CSRP2 binding protein & & (trithorax homolog, Drosophila) \\
\hline DNMT1 & DNA (cytosine-5-)-methyltransferase 1 & MLL3 & Myeloid/lymphoid or mixed-lineage leukemia 3 \\
\hline DNMT3A & DNA (cytosine-5-)-methyltransferase $3 \alpha$ & MLL5 & Myeloid/lymphoid or mixed-lineage leukemia 5 \\
\hline DNMT3B & DNA (cytosine-5-)-methyltransferase $3 \beta$ & & (trithorax homolog, Drosophila) \\
\hline \multirow[t]{2}{*}{ DOT1L } & DOT1-like, histone H3 methyltransferase & MYSM1 & Myb-like, SWIRM and MPN domains 1 \\
\hline & (S. cerevisiae) & KAT8 & $\mathrm{K}$ (lysine) acetyltransferase 8 \\
\hline DZIP3 & DAZ interacting protein 3 , zinc finger & KAT7 & $\mathrm{K}$ (lysine) acetyltransferase 7 \\
\hline \multirow[t]{2}{*}{ EHMT2 } & Euchromatic histone-lysine & KAT6A & $\mathrm{K}$ (lysine) acetyltransferase $6 \mathrm{~A}$ \\
\hline & N-methyltransferase 2 & KAT6B & $\mathrm{K}$ (lysine) acetyltransferase 6B \\
\hline \multirow[t]{2}{*}{ ESCO1 } & Establishment of cohesion 1 homolog 1 & NCOA1 & Nuclear receptor coactivator 1 \\
\hline & (S. cerevisiae) & NCOA3 & Nuclear receptor coactivator 3 \\
\hline \multirow[t]{2}{*}{ ESCO2 } & Establishment of cohesion 1 homolog 2 & NCOA6 & Nuclear receptor coactivator 6 \\
\hline & (S. cerevisiae) & NEK6 & NIMA (never in mitosis gene a)-related kinase 6 \\
\hline HAT1 & Histone acetyltransferase 1 & NSD1 & Nuclear receptor binding SET domain protein 1 \\
\hline HDAC1 & Histone deacetylase 1 & PAK1 & P21 protein $(\mathrm{Cdc} 42 / \mathrm{Rac})$-activated kinase 1 \\
\hline HDAC10 & Histone deacetylase 10 & PRMT1 & Protein arginine methyltransferase 1 \\
\hline HDAC11 & Histone deacetylase 11 & PRMT2 & Protein arginine methyltransferase 2 \\
\hline HDAC2 & Histone deacetylase 2 & PRMT3 & Protein arginine methyltransferase 3 \\
\hline HDAC3 & Histone deacetylase 3 & PRMT5 & Protein arginine methyltransferase 5 \\
\hline HDAC4 & Histone deacetylase 4 & PRMT6 & Protein arginine methyltransferase 6 \\
\hline HDAC5 & Histone deacetylase 5 & PRMT7 & Protein arginine methyltransferase 7 \\
\hline HDAC6 & Histone deacetylase 6 & PRMT8 & Protein arginine methyltransferase 8 \\
\hline HDAC7 & Histone deacetylase 7 & RNF2 & Ring finger protein 2 \\
\hline HDAC8 & Histone deacetylase 8 & RNF20 & Ring finger protein 20 \\
\hline HDAC9 & Histone deacetylase 9 & SUV39H1 & Suppressor of variegation 3-9 homolog 1 \\
\hline \multirow{2}{*}{$\begin{array}{l}\text { KDM5B } \\
\text { RPS6KA3 }\end{array}$} & Lysine (K)-specific demethylase 5B & & (Drosophila) \\
\hline & $\begin{array}{l}\text { Ribosomal protein } \mathrm{S} 6 \text { kinase, } 90 \mathrm{kDa} \text {, } \\
\text { polypeptide } 3\end{array}$ & SUV420H1 & $\begin{array}{l}\text { Suppressor of variegation } 4-20 \text { homolog } 1 \\
\text { (Drosophila) }\end{array}$ \\
\hline RPS6KA5 & $\begin{array}{l}\text { Ribosomal protein } \mathrm{S} 6 \text { kinase, } 90 \mathrm{kDa} \text {, } \\
\text { polypeptide } 5\end{array}$ & UBE2A & Ubiquitin-conjugating enzyme E2A \\
\hline SETD1A & SET domain containing $1 \mathrm{~A}$ & UBE2B & Ubiquitin-conjugating enzyme E2B \\
\hline SETD1B & $\begin{array}{l}\text { SET domain containing } 1 \mathrm{~B} \\
\text { SET domain containing IA }\end{array}$ & USP16 & Ubiquitin specific peptidase 16 \\
\hline SETD2 & $\begin{array}{l}\text { SET domain containing } 1 \mathrm{~B} \\
\text { SET domain containing } 2\end{array}$ & USP2 1 & Ubiquitin specific peptidase 21 \\
\hline & $\begin{array}{l}\text { SET domain containıng } 2 \\
\text { SET domain containing } 3\end{array}$ & USP22 & Ubiquitin specific peptidase 22 \\
\hline SETD4 & $\begin{array}{l}\text { SE } 1 \text { domain containing } 3 \\
\text { SET domain containing } 4\end{array}$ & WHSC1 & Wolf-Hirschhorn syndrome candidate 1 \\
\hline SETD4 & SE1 domain containing 4 & $\mathrm{~B} 2 \mathrm{M}$ & $\beta$-2-microglobulin \\
\hline $\begin{array}{l}\text { SETD5 } \\
\text { SETD6 }\end{array}$ & $\begin{array}{l}\text { SET domain containıng } 5 \\
\text { SET domain containing } 6\end{array}$ & HPRT1 & Hypoxanthine phosphoribosyltransferase 1 \\
\hline $\begin{array}{l}\text { SETD6 } \\
\text { SETD7 }\end{array}$ & $\begin{array}{l}\text { SET domain containıng } 6 \\
\text { SET domain containing (lvsine }\end{array}$ & RPL13A & Ribosomal protein L13a \\
\hline & methyltransferase) 7 & GAPDH & Glyceraldehyde-3-phosphate dehydrogenase \\
\hline SETD8 & SET domain containing (lysine & ACTB & Actin, $\beta$ \\
\hline SEIDO & methyltransferase) 8 & HGDC & Human genomic DNA contamination \\
\hline
\end{tabular}

Table I. Continued. 


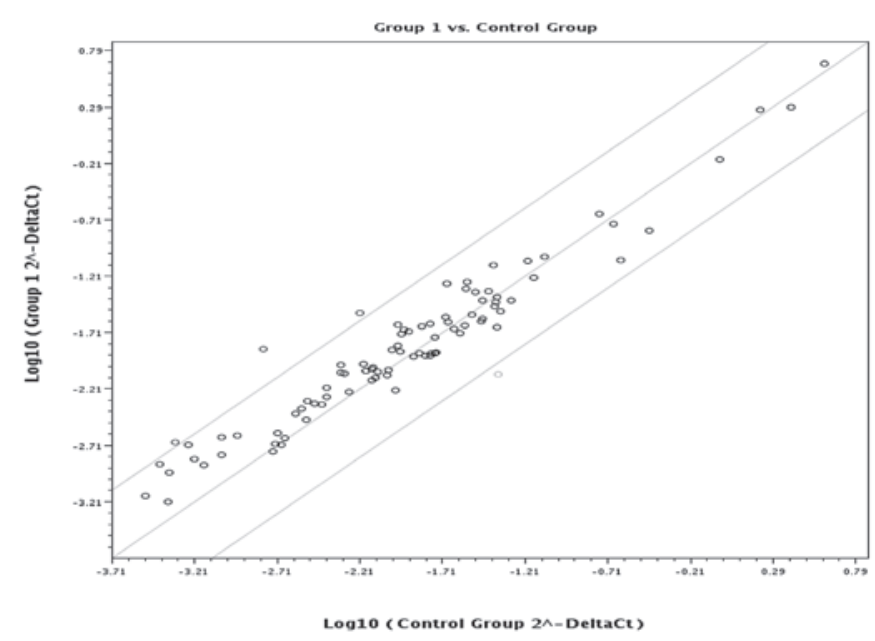

Figure 1. Scatter plot of the log base 10 of the hybridization intensity of each gene in the two groups [x-axis, control group; y-axis, EAC (Group 1) group]. The middle line indicates a fold-change $\left(2^{-\Delta \mathrm{Ct}}\right)$ of 1 . The top and the bottom lines indicate the desired fold-change in gene expression threshold. The three points above the line at the top represent upregulated (AURKA, AURKB, NEK6) genes. The one point under the bottom line represents a downregulated (MBD2) gene.

PCR array is a 96-well plate containing $\mathrm{RT}^{2}$ Profiler $^{\mathrm{TM}} \mathrm{PCR}$ Primer Assays for a set of 84 related genes, plus five housekeeping genes and three controls.

Data analysis. Data were analyzed using $\mathrm{RT}^{2}$ profiler PCR array data analysis software (http://www.sabiosciences.com/ pcrarraydataanalysis.php). The website also allowed online analysis. For each PCR reaction, the Excel sheet calculated two normalized average cycle threshold $(\mathrm{Ct})$ values, a paired $\mathrm{t}$-test $\mathrm{p}$-value and a fold-change. PCR array quantification was based on the $\mathrm{Ct}$ number. A gene was considered not detectable when $\mathrm{Ct}>32$. Ct was defined as 35 for the $\Delta \mathrm{Ct}$ calculation when the signal was under detectable limits.

Fold-change and fold-regulation values $>2$ were indicative of upregulated gene; fold-change values $<0.5$ and fold-regulation values $<-2$ were indicative of downregulated genes.

Statistics. Data were statistically analyzed with $\mathrm{RT}^{2}$ profiler PCR array data analysis software (http://www.sabiosciences. com/pcrarraydataanalysis.php). Results were expressed as the mean values \pm standard deviation and the $p$-values were calculated based on a Student's t-test of the replicate $2^{-\Delta \mathrm{Ct}}$ values for each gene in the control group, esophageal adenocarcinoma group and erosive esophagitis group. A p-value $<0.05$ was accepted as statistically significant.

\section{Results}

A total of 60 patients were divided into three groups: 20 patients as a control group (who had normal esophageal mucosa with no esophagogastroduodenoscopic or histopathological lesions), 20 patients with EE without Barrett's esophagus and microscopic adenocarcinoma and 20 patients with macroscopic and histopathological adenocarcinoma. The mean age \pm SD of the control group was $51.5 \pm 11.3$ years. The mean age \pm SD of the

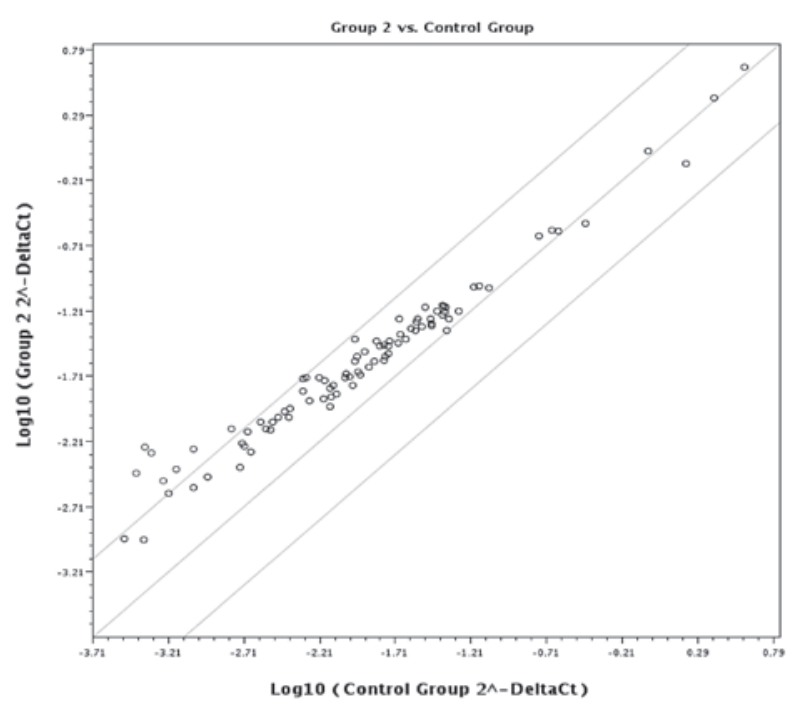

Figure 2. Scatter plot of the log base 10 of the hybridization intensity of each gene in the two groups [x-axis, control group; y-axis, EE (Group 2) group]. The middle line indicates a fold-change $\left(2^{-\Delta \mathrm{Ct}}\right)$ of 1 . The top and the bottom lines indicate the desired fold-change in gene expression threshold. The seven points above the top line represent upregulated (AURKA, AURKC, HDAC9, NEK6, HDAC8, SETD5, SETD7) genes.

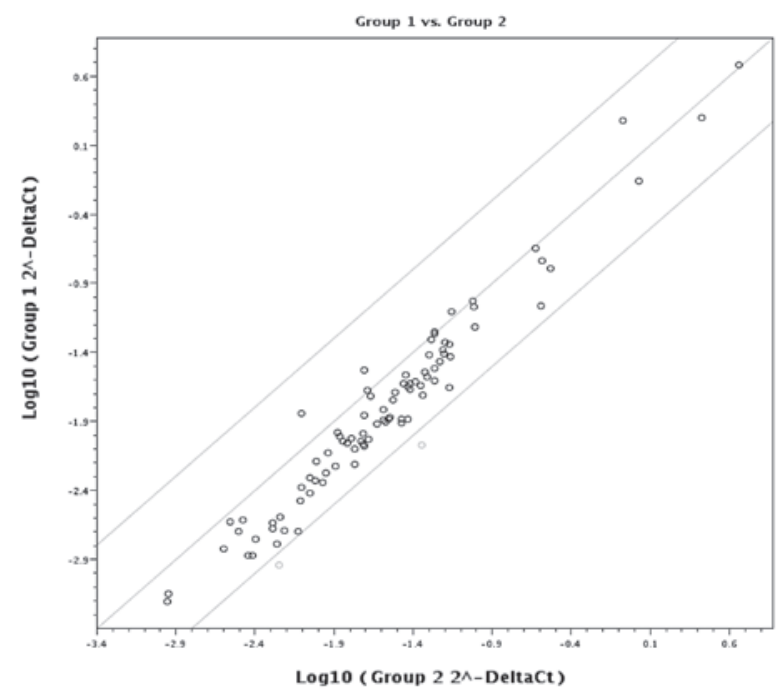

Figure 3. Scatter plot of the log base 10 of the hybridization intensity of each gene in the two groups [x-axis, EAC (group 1); y-axis, the EE (group 2) group]. The middle line indicates a fold-change $\left(2^{-\mathrm{ACt}}\right)$ of 1 . The top and the bottom lines indicate the desired fold-change in gene expression threshold. The two points under the bottom line represent downregulated (MBD2, MSYM1) genes.

EE group was $56.6 \pm 10.2$ years. The mean age \pm SD of the EAC group was $58.6 \pm 12.4$ years.

For the three groups genetic analysis was used to investigate the expression of 84 key genes (Table I) encoding enzymes known or predicted to modify genomic DNA and histones to regulate chromatin accessibility and therefore gene expression.

Upregulated and downregulated genes in the EAC and control group are summarized in Table II. AURKA, AURKB, NEK6 were expressed at significantly higher levels in the EAC than in the control group. MBD2 was expressed significantly 
Table II. Differentially upregulated and downregulated genes between the esophageal adenocarcinomas and control group.

\begin{tabular}{lccr}
\hline & Fold-change & $95 \%$ CI & p-value \\
\hline Upregulated genes & & & \\
AURKA & 2.1809 & $(0.74-3.62)$ & 0.041697 \\
AURKB & 2.5729 & $(1.33-3.81)$ & 0.004832 \\
NEK6 & 8.6782 & $(1.79-15.56)$ & 0.002312 \\
Downregulated genes & & & \\
$\quad$ MBD2 & 0.3682 & $(0.20-0.54)$ & 0.000193 \\
Housekeping genes & & & \\
for internal control & & & 0.0681 \\
HPRT1 & 1.3076 & $(0.87-1.74)$ & 0.310487 \\
RPL13A & 0.7333 & $(0.49-0.97)$ & 0.1399 \\
GAPDH & 1.1842 & $(0.81-1.56)$ & 0.56202 \\
ACTB & 0.7797 & $(0.47-1.08)$ & 0.22952 \\
HGDC & 2.6061 & $(0.00001-5.70)$ & \\
\hline
\end{tabular}

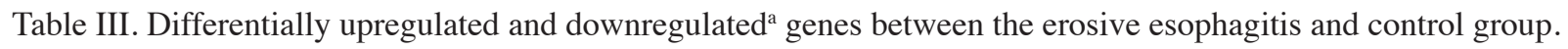

\begin{tabular}{lccc}
\hline & Fold-change & $95 \%$ CI & p-value \\
\hline Upregulated genes & & & \\
AURKA & 3.5414 & $(0.86-6.23)$ & 0.024265 \\
AURKC & 5.3826 & $(0.00001-10.99)$ & 0.040191 \\
HDAC9 & 10.676 & $(0.00001-24.29)$ & 0.036345 \\
NEK6 & 4.771 & $(0.55-8.99)$ & 0.025135 \\
HDAC8 & 2.3888 & $(0.79-3.98)$ & 0.052014 \\
SETD5 & 2.0724 & $(0.70-3.45)$ & 0.100915 \\
SETD7 & 2.492 & & 0.8001 \\
Housekeeping genes & & & \\
for internal control & & $(1.16-3.83)$ & 0.084585 \\
HPRT1 & 1.4659 & $(0.89-1.38)$ & 0.615362 \\
RPL13A & 1.1343 & $(0.87-1.40)$ & 0.154067 \\
GAPDH & 1.1331 & $(0.69-1.41)$ & 0.571422 \\
ACTB & 1.0518 & $(0.00001-29.89)$ & 0.05754 \\
HGDC & 12.8065 & & \\
\hline
\end{tabular}

${ }^{a}$ No differentially downregulated genes were noted between the two groups. CI, confidence interval.

lower in the EAC than in the control group. Fig. 1 is a scatter plot of the log base 10 of the hybridization intensity of each gene in the two groups [x-axis, control group; y-axis, the EAC (group 1) group]. The middle line indicates a fold-change $\left(2^{-\Delta \mathrm{Ct}}\right)$ of 1 . The top and the bottom lines indicate the desired fold-change in gene expression threshold. Expression of 80 key genes was unchanged, showing no significant difference in expression between the two groups. The three points above the top line indicate upregulated (AURKA, AURKB, NEK6) genes. The one point under the bottom line represents a downregulated (MBD2) gene.

Upregulated and downregulated genes in the EE and control group are summarized in Table III. Seven genes (AURKA,
AURKC, HDAC9, NEK6, HDAC8, SETD5, SETD7) were upregulated and AURKA, AURKC, HDAC9, NEK6 were expressed at significantly higher levels in EE than in the control group. There were no downregulated genes in the two groups. Fig. 2 is a scatter plot of the log base 10 of the hybridization intensity of each gene in the two groups [x-axis, control group; y-axis, EE (group 2) group]. The middle line indicates a fold-change $\left(2^{-\Delta \mathrm{Ct}}\right)$ of 1 . The top and the bottom lines indicate the desired fold-change in gene expression threshold. Expression of 77 key genes was unchanged with no significant difference in gene expression between the two groups. The seven points above the top line represent upregulated (AURKA, AURKC, HDAC9, NEK6, HDAC8, SETD5, SETD7) genes. 
Table IV. Differentially upregulated ${ }^{\mathrm{a}}$ and downregulated genes between esophageal adenocarcinoma and erosive esophagitis

\begin{tabular}{lccc}
\hline & Fold-change & $95 \%$ CI & p-value \\
\hline Downregulated & & & \\
MBD2 & 0.3849 & $(0.17-0.60)$ & 0.008897 \\
MYSM1 & 0.3418 & $(0.12-0.57)$ & 0.178543 \\
Housekeeping genes & & & \\
(for internal control) & & & 0.984553 \\
HPRT1 & 1.0676 & $(0.42-0.96)$ & 0.290211 \\
RPL13A & 0.6926 & $(0.68-1.41)$ & 0.561437 \\
GAPDH & 1.0459 & $(0.39-0.99)$ & 0.185571 \\
ACTB & 0.6882 & $(1.18-2.58)$ & 0.221942 \\
B2M & 1.8789 & & \\
\hline
\end{tabular}

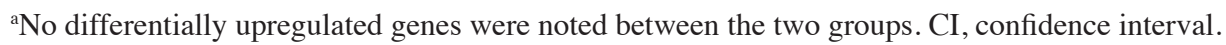

A

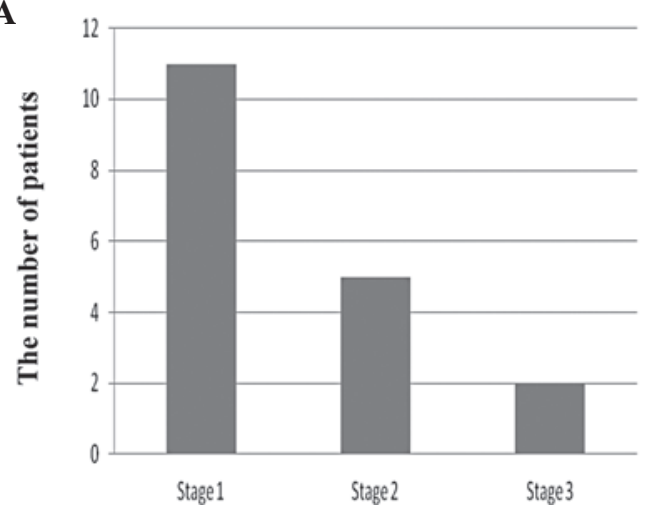

B

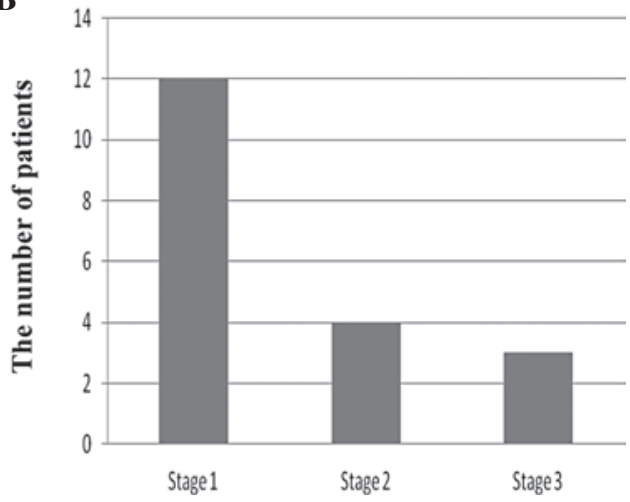

Figure 4. Correlation between the number of patients with overexpression of the (A) AURKA and (B) NEK6 genes and the stage of esophageal adenocarcinoma.

A

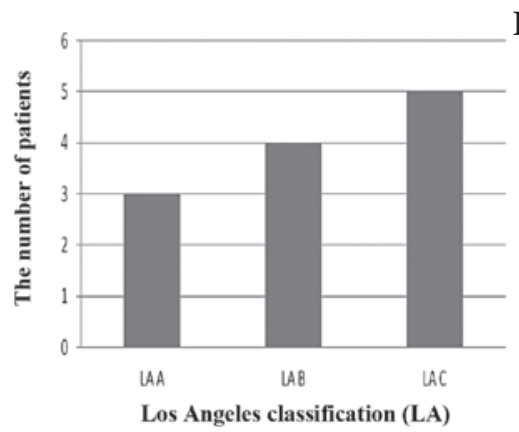

B

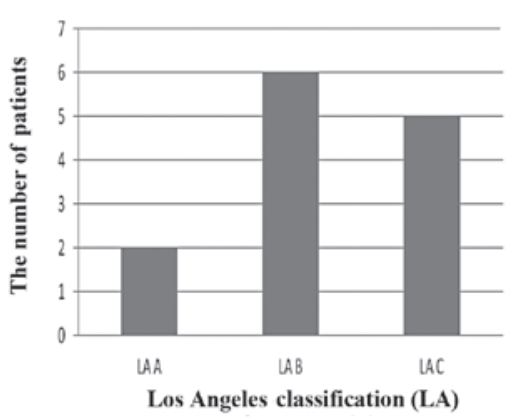

Figure 5. Correlation between the number of patients with overexpression of the (A) AURKA and (B) NEK6 genes and the Los Angeles classification of erosive esophagitis. LA A, Los Angeles classification Grade A; LA B, Los Angeles classification Grade B; LA C, Los Angeles classification Grade C.

Upregulated and downregulated genes in EAC and EE are summarized in Table IV. There was no significant difference in gene upregulation between the two groups. Two genes (MBD2 and MYSM1) were downregulated and MBD2 was significantly downregulated in EAC compared to the EE group. Fig. 3 is a plot of the log base 10 of the hybridization intensity of each gene in the two groups (x-axis represents group 1, EAC, and the y-axis shows group 2, EE). The middle line indicates a fold-change $\left(2^{-\Delta \mathrm{C} t}\right)$ of 1 . The top and bottom lines indicate the desired fold-change in gene expression threshold. The expression of 82 key genes was unchanged and no significant difference in gene upregulation was noted between the two groups. The two points under the bottom line represent downregulated (MBD2, MSYM1) genes.

The NEK6 and AURKA genes were significantly upregulated in the EAC and EE groups compared to the control group. 


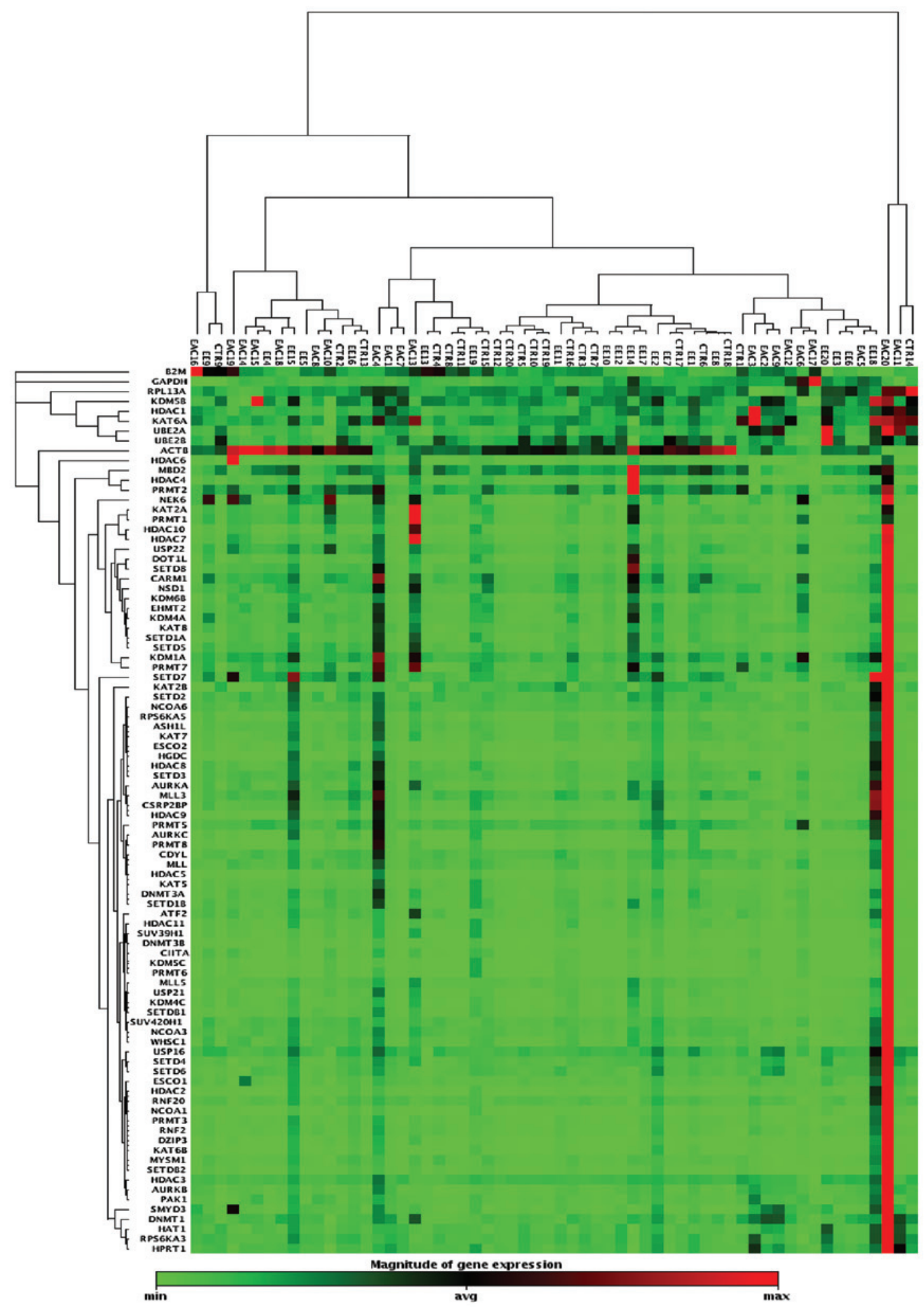

Figure 6. The clustergram creates a heat map with dendrograms to indicate genes that are co-regulated. The color saturation reflects the magnitude of the change in gene expression. Green squares represent lower gene expression in the experimental samples (ratios <1); black squares represent genes equally expressed (ratios near 1); red squares represent higher than control levels of gene expression (ratios $>1$ ); gray squares indicate insufficient or missing data. The $\mathrm{x}$-axis indicates the groups (EAC, esophageal adenocarcinoma; EE, erosive esophagitis; CTR, control group) and the y-axis indicates the genes.

The correlation between the AURKA gene and the stage of EAC is summarized in Fig. 4A. There were more patients in the stage 1 group than in the stage 2 and stage 3 groups $(\mathrm{p}<0.05)$.
The correlation between the NEK6 gene and the stage of EAC is summarized in Fig. 4B. Again, there were more patients classified in the stage 1 group than in stage 2 and stage 3 groups $(\mathrm{p}<0.05)$. 
The correlation between expression of the AURKA gene and the Los Angeles classification of erosive esophagitis are summarized in Fig. 5A. Expression of the AURKA gene was found to be elevated dependent on the grade of EE according to the Los Angeles Classification.

The correlation between expression of the NEK6 gene and the Los Angeles classification of EE is summarized in Fig. 5B. The patients with overexpression of the NEK6 gene were more prevalent in the LA Grade B than LA Grade A group ( $p>0.05$ ).

A clustergram analysis based on differentially expressed genes between the three groups is shown in Fig. 6. The clustergram creates a heat map with dendrograms to show genes that are co-regulated. The color saturation reflects the magnitude of the change in gene expression. Green squares represent lower gene expression in the experimental samples (ratios less than 1); black squares represent genes equally expressed (ratios near 1); red squares represent higher than control levels of gene expression (ratios greater than 1); gray squares indicate insufficient or missing data.

\section{Discussion}

Understanding the epigenetic structure of carcinomas provides important information on carcinogenesis. Expanding the information on the molecular biology of cancer may result in better follow-up of precancerous and cancer lesions $(13,16)$. Epigenetic changes in stem cells provide important information concerning cancer aetiology, and epigenetic alterations such as gene expression have been used as biomarkers in recent years (17).

In the present study, three genes were overexpressed in the EAC group, with AURKA, AURKB and NEK6 being significantly more highly expressed than levels in the control group. Four genes (AURKA, AURKC, HDAC9, NEK6) were significantly more highly expressed in the EE group than levels in the control group. AURKA and NEK6 genes were significantly more highly expressed in the EE group and in the EAC group than levels in the control group.

Recent studies have shown that the aurora kinase family, polo-like kinase family and NIMA (never in mitosis gene A) kinase family control cell cycle $(18,19)$. Aurora kinases are activated through autophosphorylation at the activation loop unlike most kinases within the cell (20) and NimA promotes mitotic chromosome condensation through phosphorylation of histone $\mathrm{H} 3$ at serine 10 and may compose the nuclear membrane division during mitotic exit (21).

Enzymes in the Aurora kinase family are encoded by the AURKA (also called AIK/ARK1/AURA/AURORA2/BTAK/ MGC34538/STK15/STK6/STK7) gene, which is localized on $20 q 13.2(22,23)$. These enzymes play very important roles in mitosis and meiosis for healthy cell proliferation. AURKA is a serine/threonine kinase acting as a regulator of centrosome function/duplication, mitotic entry, and bipolar spindle assembly (24). AURKA protein levels and kinase activity are low in the G1/S phase; accumulate during G2/M and decrease rapidly following mitosis (25). AURKA is an important kinase-encoding gene involved in centrosome duplication and distribution; its overexpression leads to centrosome amplification, chromosomal instability and aneuploidy in several cancer types $(26,27)$. AURKA overexpression has been found in numerous tumor cells and tissues including gastric cancer, breast cancer, colorectal cancer, bladder cancer, pancreatic cancer, ovarian cancer, prostate cancer and esophageal squamous-cell carcinoma, esophageal adenocarcinoma and Barrett's esophagus (27,28). Dar et al demonstrated overexpression of mitotic kinase encoding gene in upper gastrointestinal adenocarcinomas through the immunohistochemical analysis of 130 tumors. This overexpression was more prevalent in gastroesophageal junction adenocarcinomas and lower in esophageal, Barrett-related adenocarcinomas (BAS) than in antrum and body gastric adenocarcinomas. They also found that the expression of AURKA caused an anti-apoptotic effect in gastrointestinal cancer cells with drug-induced apoptosis in an in vitro model (27). Rugge et al found that AURKA immunostaining increased significantly along with the Barrett's carcinogenesis, from Barrett's mucosa even without metaplasia towards Barrett's adenocarcinoma (24). AURKA appears to play an important role in the carcinogenesis of esophageal adenocarcinoma and will be an important target for surveillance, diagnosis, treatment and prognosis in Barrett's esophagus. The positive relationship between Barrett's esophagus and AURKA is important for our results since in this study we demonstrated that AURKA is upregulated in erosive esophagitis and the AURKA gene was found to be elevated dependent on the grade of erosive esophagitis based on the Los Angeles Classification. GERD can yield to complications such as erosive esophagitis and strictures; furthermore, it can cause Barrett's esophagus, which can progress to adenocarcinoma (29). We believe that ascertaining whether the AURKA gene may be used as an early marker in erosive esophagitis toward the development of EAC is crucial.

NIMA is another gene found to be related to cell cycle dysfunction when it is overexpressed or underexpressed. NIMA (never in mitosis gene a)-related kinase 6 (NEK6; also called SID6-1512) is localized on chromosome 9q33-34 and is a serine/threonine kinase that belongs to the Neks (NIMArelated kinases) family, which has been implicated in mitosis control (30). Yin et al previously found that human Nek6 is required for metaphase-anaphase transition during cell cycle progression (31). It is believed that interfering with Nek6 function causes mitotic arrest and triggers apoptosis $(32,33)$. The negative mutant form of Nek6 was found to induce spindle defects, abnormal chromosome segregation, mitotic arrest and apoptosis (34). Overexpression of Nek6 was shown in hepatocellular carcinoma as compared with the adjacent normal tissue as an evidence of its antiapoptotic effect (18). Overexpression of NEK6 was associated with histological grade, level of $\alpha$ feto protein and poor prognosis. NEK6 was shown to mediate human cancer cell transformation and was proposed as a potential cancer therapeutic marker in a previous study (34). Takeno et al stated that NEK6 is a potential marker of gastric cancer regardless of stage and since conventional staging of tumors are not adequate to predict individual prognosis, genetic analyses of tumor tissues may provide better opportunities to predict disease outcome for each individual and may even predict response to therapy (35). The authors selected seven focus genes showing a 2-fold change; four had not been previously evaluated for the association with gastric tumors. NEK6 was one these four new genes (35). They concluded that mapping of gene expression data on large 
sample numbers helped to identify two novel candidate genes, INHBA and NEK6, that are promising potential markers of gastric cancer. Nassirpour $e t$ al revealed that the protein level and kinase activity of Nek6 are highly elevated in a variety of malignant human cancers including breast, uterus, colon, stomach, ovary, lung, kidney, rectum, thyroid, cervix, prostate, pancreas, small intestine cancer cells, and knockdown of Nek6 resulted in reduction of tumors in a nude mouse xenograft model (34). They concluded that since inhibition of NEK6 specifically induces cell death in tumor cells and not in normal tissues, NEK6 inhibitors are a better therapeutic option with lower side effects than cytotoxic antitumor agents.

This is the first study investigating the impact of NEK6 in esophageal adenocarcinoma. Our data show the significant upregulation of NEK6 in erosive esophagitis and esophageal adenocarcinoma. NEK6 was more prevalent in samples with Los Angeles classification B than A in erosive esophagitis demonstrating that NEK6 and AURKA are more evident in more severe forms of esophagitis.

Erosive esophagitis is chronic damage of the esophagus caused by acid, pepsin and biliary salts. Environmental insults cause genetic and epigenetic alterations and they affect the expression of tumor-progenitor genes. Chronic injury is a major cause of cancer even though it is not inherently mutagenic (16). There are studies with large number of patients and long followup periods for GERD patients investigating whether they are at risk of developing esophageal adenocarcinoma. In a Swedish nationwide case-control study, gastroesophageal reflux and obesity were identified as strong and independent risk factors for esophageal adenocarcinoma. The risk increased with duration and severity of reflux symptoms and with increasing body mass index (36). Erichsen et al performed a nationwide cohort study in Denmark using data from 33,849 GERD patients and concluded that erosive but not non-erosive reflux disease has an impact on the development of adenocarcinoma emphasizing inflammation as an important factor in carcinogenesis (37).

In our study, in addition to AURKA and NEK6, HDAC9 and AURKC were significantly upregulated in EE compared to the control group. These genes were not expressed in EAC. Wu et al stated that AURKC was overexpressed in inflamed cervical tissue specimens and HDAC inhibitors are therapeutic for several inflammatory conditions $(38,39)$. Do these genes have a role in addition to defect of the defense mechanism of the esophagus in patients with EE in gastroesophageal disease?

MBD2 was significantly downregulated in EAC compared to $\mathrm{EE}$ and the control group. MBD2 is a member of the MBD protein family. MBD2 binds to methylated promoter $\mathrm{CpG}$ islands and acts as a methylation-dependent transcriptional repressor (40). MBD2 has a role in the activation of methylated and unmethylated genes (42). Expression of MBD2 was particularly low in brain tumors, immune thrombocytopenia and in colorectal and gastric carcinomas (42-44) as found in our study. The reasons for the loss of MBD2 expression and the functional consequences are unknown (44). Thus, MBD2 should be studied further in relation to its association with esophageal adenocarcinoma.

This is the first study concerning the epigenetic chromatin histone modification in EE and EAC patients. Compared to the other genes investigated, AURKA and NEK6 were notably upregulated in EAC and EE. AURKA was proven to be associ- ated with EAC in previous studies, and we found similar results for AURKA. This is the first study investigating the impact of NEK6 in esophageal adenocarcinoma, and our data revealed the significant upregulation of NEK6 in erosive esophagitis and esophageal adenocarcinoma. Our study is also the first study aiming to detect the presence of genetic upregulation in EE, a lesion which is not considered to be a precancerous lesion. These results pave the way for future studies with larger numbers of patients and longitudinal studies with longer followup periods. In a recent study (7), we found that low prevalence of Barrett's esophagus was demonstrated in a Western Turkish population. Based on these data, we intend to explore expression of AURKA and NEK6 genes in the future at the national level, using another group with Barrett's esophagus.

It is hoped that future studies may address the following questions: i) Is erosive esophagitis a precancerous lesion and once detected, is surveillance required? ii) Can AURKA and NEK6 be used as screening tests for esophageal adenocarcinoma in erosive esophagitis? iii) What are the ranges of AURKA and NEK6 overexpression predictive of the prognosis and the outcome of antitumor therapy? iv) Can AURKA and NEK6 be used as therapeutic targets?

In conclusion, we demonstrated overexpression of AURKA and NEK6 in erosive esophagitis and esophageal adenocarcinoma in a Turkish population. Understanding the molecular pathophysiology of the disease will aid in elucidating the steps for diagnosis, therapy and prognosis. AURKA and NEK6 are two promising genetic markers for erosive esophagitis and esophageal adenocarcinoma.

\section{Acknowledgements}

This study was supported by the Celal Bayar University Coordinator of the Scientific Research Projects (2009-053) Manisa, Turkey.

\section{References}

1. Ekiz F, Ormeci N, Coban S, et al: Association of methylenetetrahydrofolate reductase C677T-A1298C polymorphisms with risk for esophageal adenocarcinoma, Barrett's esophagus, and reflux esophagitis. Dis Esophagus: Sep 23, 2011 (Epub ahead of print).

2. Yoon $\mathrm{HH}$, Khan M, Shi Q, et al: The prognostic value of clinical and pathologic factors in esophageal adenocarcinoma: a Mayo cohort of 796 patients with extended follow-up after surgical resection. Mayo Clin Proc 85: 1080-1089, 2010.

3. Bor S, Vardar R, Ormeci N, et al: Prevalence patterns of gastric cancers in Turkey: model of a developing country with high occurrence of Helicobacter pylori. J Gastroenterol Hepatol 22: 2242-2245, 2007.

4. Reavis KM, Morris CD, Gopal DV, Hunter JG and Jobe BA: Laryngopharyngeal reflux symptoms better predict the presence of esophageal adenocarcinoma than typical gastroesophageal reflux symptoms. Ann Surg 239: 849-856, 2004.

5. Das A: Tumors of the Esophagus. In: Sleisenger and Fordtran's Gastrointestinal and Liver Disease. Feldman M, Friedman L Friedman S and Brandt JL (eds). Saunders Elsevier, Philadelphia, pp745-773, 2010 .

6. Zendehdel N, Biramijamal F, Hossein-Nezhad A, Zendehdel N, Sarie H, Doughaiemoghaddam M and Pourshams A: Role of cytochrome P450 2C19 genetic polymorphisms in the therapeutic efficacy of omeprazole in Iranian patients with erosive reflux esophagitis. Arch Iran Med 13: 406-412, 2010.

7. Bayrakçi B, Kasap E, Kitapçioğlu G and Bor S: Low prevalence of erosive esophagitis and Barrett esophagus in a tertiary referral center in Turkey. Turk J Gastroenterol 19: 145-151, 2008. 
8. Bor S, Mandiracioglu A, Kitapcioglu G, Caymaz-Bor C and Gilbert RJ: Gastroesophageal reflux in a low-income region in Turkey. Am J Gastroenterol 100: 744-754, 2005.

9. Peng D, Hu TL, Jiang A, Washington MK, Moskaluk CA, Schneider-Stock R and El-Rifai W: Location-specific epigenetic regulation of the metallothionein 3 gene in esophageal adenocarcinomas. PLoS One 6: e22009, 2011.

10. Sharma S, Kelly TK and Jones PA: Epigenetics in cancer. Carcinogenesis 31: 27-36, 2010.

11. Jones PA and Baylin SB: The fundamental role of epigenetic events in cancer. Nat Rev Genet 3: 415-428, 2002.

12. Jones PA and Baylin SBl: The epigenomics of cancer. Cell 128 : 683-692, 2007.

13. Feinberg AP, Ohlsson R and Henikoff S: The epigenetic progenitor origin of human cancer. Nat Rev Genet 7: 21-33, 2006

14. Yoo CB and Jones PA: Epigenetic therapy of cancer: past, present and future. Nat Rev Drug Discov 5: 37-50, 2006.

15. Lundell LR, Dent J, Bennett JR, et al: Endoscopic assessment of oesophagitis: clinical and functional correlates and further validation of the Los Angeles classification. Gut 45: 172-180, 1999.

16. Egger G, Liang G, Aparicio A and Jones PA: Epigenetics in human disease and prospects for epigenetic therapy. Nature 429: 457-463, 2004.

17. Tzao C, Tung HJ, Jin JS, et al: Prognostic significance of global histone modifications in resected squamous cell carcinoma of the esophagus. Mod Pathol 22: 252-260, 2009.

18. Cao X, Xia Y, Yang J, et al: Clinical and biological significance of never in mitosis gene A-related kinase 6 (NEK6) expression in hepatic cell cancer. Pathol Oncol Res 18: 201-207, 2012.

19. Malumbres $M$ and Barbacid M: Cell cycle kinases in cancer Curr Opin Genet Dev 17: 60-65, 2007.

20. Fu J, Bian M, Jiang Q and Zhang C: Roles of Aurora kinases in mitosis and tumorigenesis. Mol Cancer Res 5: 1-10, 2007.

21. Moniz L, Dutt P, Haider N and Stambolic V: Nek family of kinases in cell cycle, checkpoint control and cancer. Cell Div 6 : $18,2011$.

22. Sen S, Zhou H and White RA: A putative serine/threonine kinase encoding gene BTAK on chromosome $20 \mathrm{q} 13$ is amplified and overexpressed in human breast cancer cell lines. Oncogene 14 2195-2200, 1997

23. Reiter R, Gais P, Jütting U, et al: Aurora kinase A messenger RNA overexpression is correlated with tumor progression and shortened survival in head and neck squamous cell carcinoma. Clin Cancer Res 12: 5136-5141, 2006

24. Rugge M, Fassan M, Zaninotto G, et al: Aurora kinase A in Barrett's carcinogenesis Hum Pathol 41: 1380-1386, 2010.

25. Lo Iacono M, Monica V, Saviozzi S, Ceppi P, Bracco E, Papotti M and Scagliotti GV: Aurora Kinase A expression is associated with lung cancer histological-subtypes and with tumor de-differentiation. J Transl Med 9: 100, 2011.

26. Sillars-Hardebol AH, Carvalho B, de Wit M, et al: Identification of key genes for carcinogenic pathways associated with colorectal adenoma-to-carcinoma progression. Tumour Biol 31: 89-96, 2010.

27. Dar AA, Zaika A, Piazuelo MB, et al: Frequent overexpression of Aurora Kinase A in upper gastrointestinal adenocarcinomas correlates with potent antiapoptotic functions. Cancer 112 1688-1698, 2008.

28. El-Rifai W and Powell SM: Molecular and biologic basis of upper gastrointestinal malignancy. Gastric carcinoma. Surg Oncol Clin N Am 11: 273-291, 2002.
29. Shaheen N and Ransohoff DF: Gastroesophageal reflux, Barrett esophagus, and esophageal cancer: scientific review. JAMA 287: 1972-1981, 2002

30. Jee HJ, Kim AJ, Song N, Kim HJ, Kim M, Koh H and Yun J: Nek6 overexpression antagonizes p53-induced senescence in human cancer cells. Cell Cycle 9: 4703-4710, 2010.

31. Yin MJ, Shao L, Voehringer D, Smeal T and Jallal B: The serine/ threonine kinase Nek6 is required for cell cycle progression through mitosis. J Biol Chem 278: 52454-52460, 2003.

32. Belham C, Roig J, Caldwell JA, Aoyama Y, Kemp BE, Comb M and Avruch J: A mitotic cascade of NIMA family kinases. Nercc1/Nek9 activates the Nek6 and Nek7 kinases. J Biol Chem 278: 34897-34909, 2003.

33. O'Regan L and Fry AM: The Nek6 and Nek7 protein kinases are required for robust mitotic spindle formation and cytokinesis. Mol Cell Biol 29: 3975-3990, 2009.

34. Nassirpour R, Shao L, Flanagan P, Abrams T, Jallal B, Smeal T and Yin MJ: Nek6 mediates human cancer cell transformation and is a potential cancer therapeutic target. Mol Cancer Res 8: 717-728, 2011.

35. Takeno A, Takemasa I, Doki Y, et al: Integrative approach for differentially overexpressed genes in gastric cancer by combining largescale gene expression profiling and network analysis. Br J Cancer 99: 1307-1315, 2008.

36. Lagergren J: Increased incidence of adenocarcinoma of the esophagus and cardia. Reflux and obesity are strong and independent risk factors according to the SECC study. Lakartidningen J 97: 1950-1953, 2000 (In Swedish).

37. Erichsen R, Robertson D, Farkas DK, Pedersen L, Pohl H, Baron J and Sorensen HT: Erosive reflux disease increases risk for esophageal adenocarcinoma risk, compared with non-erosive reflux. Clin Gastroenterol Hepatol: Jan 12, 2012 (Epub ahead of print).

38. Wu SR, Li CF, Hung LY, Huang AM, Tseng JT, Tsou JH and Wang J: MCCAAT/enhancer-binding protein delta mediates tumor necrosis factor alpha-induced Aurora kinase $\mathrm{C}$ transcription and promotes genomic instability. J Biol Chem 286: 28662-28670, 2011.

39. Shakespear MR, Halili MA, Irvine KM, Fairlie DP and Sweet MJ: Histone deacetylases as regulators of inflammation and immunity. Trends Immunol 32: 335-343, 2011.

40. Berger $J$ and Bird A: Role of MBD2 in gene regulation and tumorigenesis. Biochem Soc Trans 33: 1537-1540, 2005.

41. Fujita H, Fujii R, Aratani S, Amano T, Fukamizu A and Nakajima T: Antithetic effects of MBD2a on gene regulation. Mol Cell Biol 23: 2645-2657, 2003.

42. Müller-Tidow C, Kügler K, Diederichs S, et al: Loss of expression of HDAC-recruiting methyl-CpG-binding domain proteins in human cancer. Br J Cancer 85: 1168-1174, 2001.

43. Kanai Y, Ushijima S, Nakanishi Y and Hirohashi S: Reduced mRNA expression of the DNA demethylase, MBD2, in human colorectal and stomach cancers. Biochem Biophys Res Commun 2: 962-966, 1999.

44. Chen ZP, Gu DS, Zhou ZP, et al: Decreased expression of MBD2 and MBD4 gene and genomic-wide hypomethylation in patients with primary immune thrombocytopenia. Hum Immunol 72: 486-491, 2011. 